\title{
Risk factors for porcine reproductive and respiratory syndrome outbreaks in Vietnamese small stock farms
}

\section{VM Truong ${ }^{* \dagger}$ and B Gummow ${ }^{*}$ s}

${ }^{*}$ School of Veterinary and Biomedical Sciences, James Cook University, Townsville, Queensland, 4811, Australia.

${ }^{\dagger}$ Regional Animal Health Office No. 2, Department of Animal Health of Vietnam, No. 23 Danang Street, Haiphong City, Vietnam.

Department of Production Animal Studies, Faculty of Veterinary Science, University of Pretoria, Onderstepoort, South Africa.

$\S$ Author for correspondence. Email: bruce.gummow@jcu.edu.au 
Supplementary Table 1. Sample size estimates and risk factors for an unmatched case control study in Quangninh province, Vietnam based on literature review and expert opinions about estimated proportion of PRRS unaffected households exposed to each potential risk factor with 95 confidence level, power 80, case: control ratio set at 1:1 and minimum OR detected set at 2.

\begin{tabular}{|c|c|c|c|c|c|c|c|c|c|}
\hline \multirow{2}{*}{ Risk factors } & \multicolumn{4}{|c|}{$\begin{array}{l}\text { Estimated proportion of household cases } \\
\text { with exposure to risk factors }\end{array}$} & \multicolumn{4}{|c|}{$\begin{array}{l}\text { Estimated proportion of PRRS unaffected } \\
\text { households with exposure to risk factors }\end{array}$} & \multirow{2}{*}{$\begin{array}{l}\text { Sample } \\
\text { size }\end{array}$} \\
\hline & Expert 1 & Expert 2 & Literature & Mean & Expert 1 & Expert 2 & Literature & Mean & \\
\hline \multicolumn{10}{|l|}{ 1. Pig movement } \\
\hline - Farm located close to the main roads & & & 65.7 & 65.7 & & & 42.9 & 42.9 & 576 \\
\hline - Farm located close to the local market or livestock market & & & 28.6 & 28.6 & & & 7.1 & 7.1 & 658 \\
\hline - $\quad$ Farm located close to a pig slaughterhouse & & & 2.9 & 2.9 & & & 8.6 & 8.6 & 694 \\
\hline \multicolumn{10}{|l|}{ 2. People contact } \\
\hline - Pig traders & & & 25.7 & 25.7 & & & 3.6 & 3.6 & 1,512 \\
\hline - Veterinary practitioners & 65.0 & 30.0 & & 47.5 & 20.0 & 40.0 & & 30.0 & 306 \\
\hline - Neighbors & 75.0 & 70.0 & & 72.5 & 50.0 & 50.0 & & 50.0 & 238 \\
\hline - Feed suppliers & 40.0 & 65.0 & & 52.5 & 30.0 & 45.0 & & 37.5 & 290 \\
\hline - Family member visit other PRRS affected farms & 35.0 & 70.0 & & 52.5 & 25.0 & 45.0 & & 35.0 & 234 \\
\hline \multicolumn{10}{|l|}{ 3. Animal contact } \\
\hline - Households keep pigs with other animals & & & 57.1 & 57.1 & & & 46.4 & 46.4 & 234 \\
\hline - $\quad$ Presence of wild animals (bird, rodent) & & & 91.0 & 91.0 & & & & 82.0 & 618 \\
\hline \multicolumn{10}{|l|}{ 4. Herd management } \\
\hline - New introduction without quarantine & 80.0 & 70.0 & & 75.0 & 70.0 & 20.0 & & 45.0 & 288 \\
\hline - Purchase of pigs from other places & 10.0 & 35.0 & & 22.5 & 20.0 & 55.0 & & 37.5 & 232 \\
\hline - Purchase of pigs from a livestock market & & & 62.9 & 62.9 & & & 30.4 & 30.4 & 304 \\
\hline - Purchase of semen for AI & 25.0 & 40.0 & & 32.5 & 20.0 & 55.0 & & 37.5 & 290 \\
\hline - Share boar with other sows & 10.0 & 55.0 & & 32.5 & 10.0 & 45.0 & & 27.5 & 316 \\
\hline - All in/all out policy & 10.0 & 30.0 & & 20.0 & 10.0 & 50.0 & & 30.0 & 246 \\
\hline
\end{tabular}




\section{Supplementary Information}

\begin{tabular}{|c|c|c|c|c|c|c|c|c|c|}
\hline \multirow{2}{*}{ Risk factors } & \multicolumn{4}{|c|}{$\begin{array}{l}\text { Estimated proportion of household cases } \\
\text { with exposure to risk factors }\end{array}$} & \multicolumn{4}{|c|}{$\begin{array}{l}\text { Estimated proportion of PRRS unaffected } \\
\text { households with exposure to risk factors }\end{array}$} & \multirow{2}{*}{$\begin{array}{l}\text { Sample } \\
\text { size }\end{array}$} \\
\hline & Expert 1 & Expert 2 & Literature & Mean & Expert 1 & Expert 2 & Literature & Mean & \\
\hline - Cooked & 20.0 & 40.0 & & 30.0 & 80.0 & 50.0 & & 65.0 & 360 \\
\hline - Uncooked & 80.0 & 55.0 & & 67.5 & 20.0 & 45.0 & & 32.5 & 298 \\
\hline \multicolumn{10}{|l|}{ 6. Hygiene } \\
\hline - Poor hygiene & 80.0 & 70.0 & & 75.0 & 75.0 & 30.0 & & 52.5 & 302 \\
\hline - Good hygiene & 5.0 & 20.0 & & 12.5 & 10.0 & 60.0 & & 35.0 & 294 \\
\hline - Effluent treatment & 2.0 & 35.0 & & 18.5 & 5.0 & 60.0 & & 32.5 & 298 \\
\hline - No effluent treatment & 75.0 & 45.0 & & 60.0 & 70.0 & 30.0 & & 50.0 & 296 \\
\hline - Disinfecting periodically & 2.0 & 30.0 & & 16.0 & 10.0 & 35.0 & & 22.5 & 350 \\
\hline - No disinfection & 90.0 & 70.0 & & 80.0 & 10.0 & 55.0 & & 32.5 & 298 \\
\hline Mean & & & & & & & & & 397 \\
\hline Median & & & & & & & & & 298 \\
\hline Mode & & & & & & & & & 298 \\
\hline
\end{tabular}


Supplementary Information

Truong et al.

New Zealand Veterinary Journal Vol(Issue), year

S4

DOI: bttp:/ / dx.doi.org/ 10.1080/00480169.2014.888640

Supplementary Table 2. Hierarchical models constructed to select risk factors associated with PRSS infected households in Yenhung district, Quangninh province, Vietnam, 2010 based on results from the univariate logistic regressions (Number of households =300).

\begin{tabular}{|c|c|c|c|c|c|c|c|c|}
\hline \multirow[b]{2}{*}{ Explanatory variables } & \multirow[b]{2}{*}{ Category } & \multirow{2}{*}{$\begin{array}{l}\text { Odds } \\
\text { Ratio }\end{array}$} & \multicolumn{2}{|c|}{ 95\% C.I. } & \multirow{2}{*}{ Coefficient } & \multirow{2}{*}{ S.E. } & \multirow{2}{*}{$\begin{array}{c}\text { Z- } \\
\text { Statistic }\end{array}$} & \multirow{2}{*}{$\begin{array}{c}\text { P- } \\
\text { Value }\end{array}$} \\
\hline & & & LCI & HCI & & & & \\
\hline \multicolumn{9}{|c|}{ Model 1: Location of the farms } \\
\hline \multirow{3}{*}{$\begin{array}{l}\text { Distance from the pen to } \\
\text { a pig abattoir }\end{array}$} & $<500 \mathrm{~m}(\mathrm{DP} 1)$ & 4.22 & 1.68 & 10.57 & 1.44 & 0.47 & 3.08 & 0.0021 \\
\hline & $501-1000 \mathrm{~m}(\mathrm{DP} 2)$ & 2.28 & 1.15 & 4.50 & 0.83 & 0.35 & 2.38 & 0.017 \\
\hline & $>1000 \mathrm{~m}$ & Reference & & & & & & \\
\hline \multicolumn{9}{|c|}{ Model 2: Farm management } \\
\hline \multirow{2}{*}{$\begin{array}{l}\text { Introduction of newly } \\
\text { purchased pigs without } \\
\text { isolation (IP) }\end{array}$} & Yes (IPY) & 2.60 & 1.08 & 6.26 & 0.96 & 0.45 & 2.14 & 0.032 \\
\hline & No (IPN) & Reference & & & & & & \\
\hline \multirow{2}{*}{$\begin{array}{l}\text { Using water from } \\
\text { irrigation system for pig } \\
\text { raising }\end{array}$} & Yes (WIRRY) & $21.46(-)$ & 11.26 & 40.88 & 3.06 & 0.33 & 9.32 & $<0.0001$ \\
\hline & No (WIRRN) & Reference & & & & & & \\
\hline \multicolumn{9}{|c|}{ Model 3: People and animal contact } \\
\hline \multirow{2}{*}{$\begin{array}{l}\text { No confinement of pet } \\
\text { animals (CONFN) }\end{array}$} & Yes & 2.51 & 1.13 & 5.58 & 0.92 & 0.41 & 2.27 & 0.023 \\
\hline & No & & & & & & & \\
\hline \multirow{2}{*}{$\begin{array}{l}\text { Family member visited } \\
\text { other PRRS affected } \\
\text { farms }\end{array}$} & Yes (OUTBY) & 3.93 & 2.20 & 7.00 & 1.37 & 0.30 & 4.64 & $<0.0001$ \\
\hline & No (OUTBN) & Reference & & & & & & \\
\hline
\end{tabular}




\section{Supplementary Information}

Truong et al.

New Zealand Veterinary Journal Vol(Issue), year

S5

DOI: http:// dx.doi.org/10.1080/00480169.2014.888640

Supplementary Figure 1. Location of Quangninh province and Yenhung district in Northern Vietnam. 
Supplementary Information

Truong et al.

New Zealand Veterinary Journal Vol(Issue), year

S6

DOI: http:/ / dx.doi.org/10.1080/00480169.2014.888640

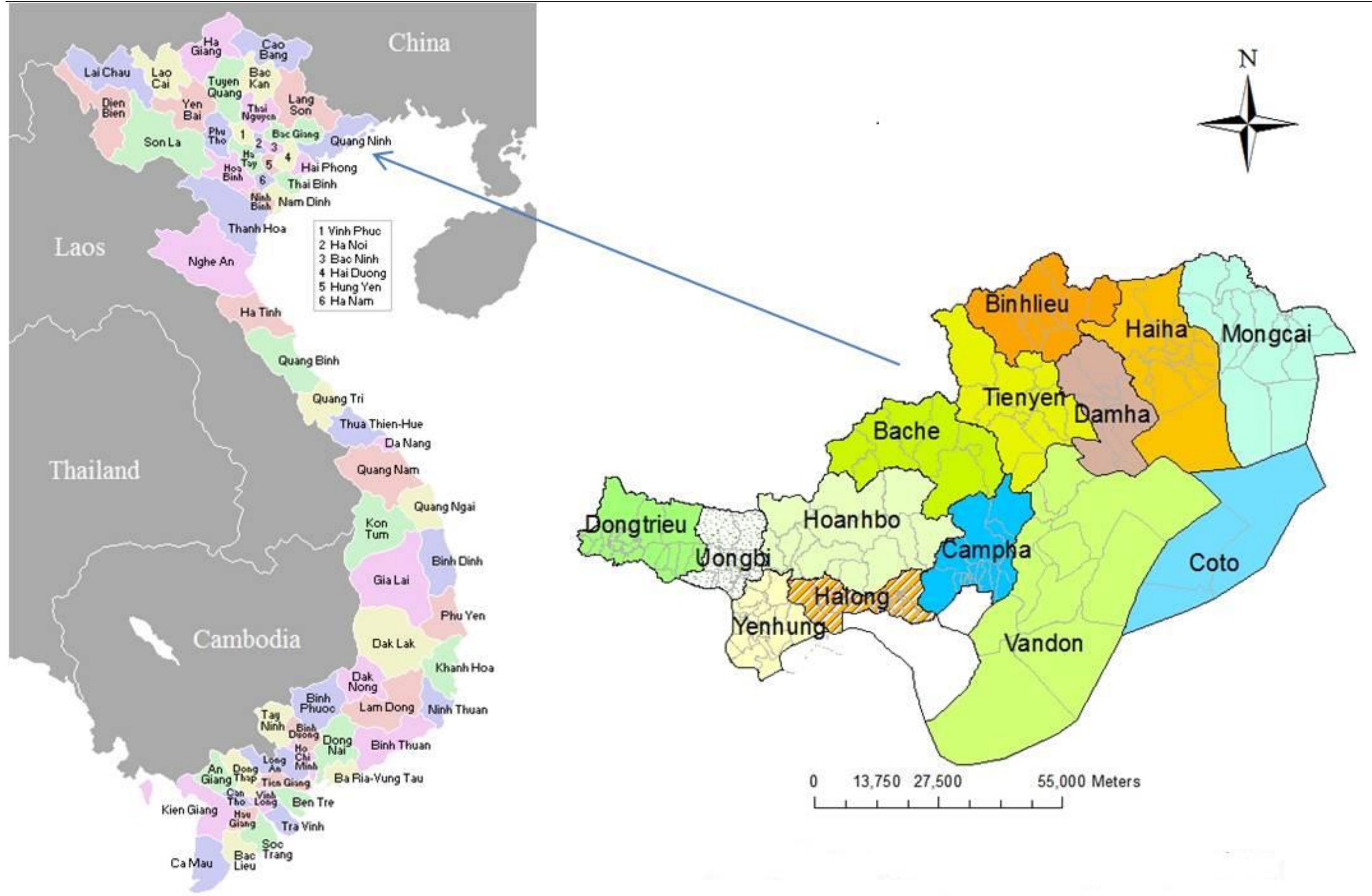




\section{Supplementary Information}




\section{Supplementary Questionnaire}

NB! This questionnaire applies only to April-June period, 2010!

\begin{tabular}{|c|l|c|}
\hline 1. & General information & \\
\hline 1.1 & Date (dd-mm-yyyy) & - \\
\hline 1.2 & Full name of pig owner (Capital letter) & \\
\hline 1.3 & Address (House No, village) & \\
\hline 1.4 & Phone number (if available) & \\
\hline 1.5 & Village (Capital letter) & \\
\hline 1.6 & Commune (Capital letter) & \\
\hline
\end{tabular}

\subsection{District (Capital letter)}

1.8 Full name of interviewer (Capital letter)

\section{Herd information (Please tick in the appropriate box)}

\subsection{Farm type}

Commercial farm

\subsection{What kind of pigs did you raise?}

\begin{tabular}{|l|l|l|l|l|l|}
\hline Young pig (10 to $20 \mathrm{kgs})$ & & $\ldots \ldots .$. & Sow & & $\ldots \ldots \ldots$ \\
\hline Finishing (over $20 \mathrm{~kg})$ & & $\ldots \ldots \ldots .$. & Boar & & $\ldots \ldots \ldots$. \\
\hline
\end{tabular}

\subsection{Herd size}

How many pigs in total did you raise?

\section{Information about premise.}

\begin{tabular}{|c|c|c|c|c|}
\hline $\begin{array}{l}\text { 3.1. How many pens? } \\
\text {............... }\end{array}$ & & Min & Most likely & $\operatorname{Max}$ \\
\hline 3.2. What kind of floor is it? & 3.3. How large is each pen $\left(\mathrm{m}^{2}\right)$ ? & & & \\
\hline Concrete & 3.4. How many pigs per pen? & & & \\
\hline Soil & 3.5. How many feeders per pen? & & & \\
\hline Partially slated & 3.6. How many drinkers per pen? & & & \\
\hline
\end{tabular}

3.7. How far is the pen from your living room?

3.8. How far is the pen from the main roads $(*)$ ?

$$
\begin{array}{|l|}
\hline<10 \mathrm{~m} \\
\hline \text { From } 11 \text { to } 20 \mathrm{~m} \\
\hline \text { Over } 20 \mathrm{~m} \text { (details) } \\
\hline
\end{array}
$$

\begin{tabular}{|c|c|c|c|c|}
\hline \multicolumn{2}{|c|}{ 3.9. What kind of breed? } & Min & Most likely & Max \\
\hline Local breed & How many? & & & \\
\hline
\end{tabular}$$
<100 \mathrm{~m}
$$

(*) Main roads are defined as national roads, district roads or commune roads 


\section{Supplementary Information}

\begin{tabular}{|c|c|c|c|c|}
\hline Hybrid breed (localxforeign breed) & How many? ...................... & ............ & ............ & . \\
\hline Foreign breed & How many? ...................... & ............ & ............ & ............ \\
\hline
\end{tabular}

Notes: - Local breed is defined as Mongcai breed

- Hybrid breed is formed by crossing local breed with foreign breed

- $\quad$ Foreign breed is defined as Landrace, Duroc, Large White, Yorkshire or crossbred between them.

\subsection{Gender}

No of males

No of females

3.4. Is there an abattoire in your farm?

3.14. Is there local market near your farm?

\begin{tabular}{|c|c|}
\hline Yes & No \\
\hline 3.5. If $y e s$, what animal are slaughtered?
\end{tabular}

3.5. If yes, what animal are slaughtered? Yes No

\begin{tabular}{|l}
\hline 3.6. How far from the pen? \\
\hline Within $500 \mathrm{~m}$ \\
\hline From $1000 \mathrm{~m}$ to $2000 \mathrm{~m}$ \\
\hline
\end{tabular}

3.15. If yes, how far is from your home?

\section{Herd management, hygiene, feed.}

\begin{tabular}{|c|c|c|c|}
\hline \multicolumn{2}{|c|}{ 4.1. Is the pen solid and liquid wastes treated by } & \multicolumn{2}{|c|}{ 4.2. How often is the pen/farm disinfected? } \\
\hline Biogas plant & & \multicolumn{2}{|l|}{ Daily } \\
\hline Taken far away from the pen & & \multicolumn{2}{|l|}{ 2-3 times/week } \\
\hline Untreated & & \multicolumn{2}{|l|}{ Never } \\
\hline Other (details) & ..................... & & \\
\hline \multicolumn{2}{|c|}{ 4.3. How often do you clean the pen, feeder, drinker } & \multicolumn{2}{|c|}{ 4.4. How do you process the feed? } \\
\hline Daily & & \multicolumn{2}{|l|}{ Buy commercial feed } \\
\hline 2-3 times/week & & \multicolumn{2}{|c|}{ Self process from agri-products } \\
\hline Sometimes & & \multicolumn{2}{|c|}{ Swill from kitchen, restaurant? } \\
\hline Never & & \multicolumn{2}{|c|}{ Cooked } \\
\hline & & \multicolumn{2}{|l|}{ Not cooked } \\
\hline \multicolumn{2}{|c|}{ 4.5. Do you apply all in/all out policy in your farm? } & \multicolumn{2}{|c|}{$\begin{array}{l}\text { 4.6. Do you seperate newly bought pigs in an } \\
\text { isolation zone before intergrating into the } \\
\text { herd? }\end{array}$} \\
\hline Yes & & Yes & \\
\hline No & & If yes, for how long? & .................... \\
\hline
\end{tabular}




\section{Supplementary Information}

4.7. Your pigs were totally confined?

\begin{tabular}{|l|l|} 
Yes & No \\
\hline No & \\
\hline
\end{tabular}

4.8. Where did you buy your breeding pigs?

\begin{tabular}{|l|l|l|l|}
\hline From the livestock market & From village where you live & \\
\hline Homebred raising pig & & From other places (details) & \\
\hline
\end{tabular}

\section{Health management}

\subsection{During April-June, did your pigs exhibit the following clinical signs:}

\begin{tabular}{|c|c|c|c|c|c|c|c|}
\hline & $<33 \%$ & $34-66 \%$ & $>66 \%$ & & $<33 \%$ & $34-66 \%$ & $>66 \%$ \\
\hline High fever $\left(\geq 40.5^{\circ} \mathrm{C}\right)$ & & & & Abortion & & & \\
\hline Anorexia & & & & Stillbirths & & & \\
\hline Red discolorations & & & & Weak piglets & & & \\
\hline $\begin{array}{l}\text { Blood spot in the } \\
\text { body }\end{array}$ & & & & Other (details)............. & & & \\
\hline Blue ears & & & & Number of death & & & \\
\hline Lethargy & & & & & & & \\
\hline
\end{tabular}

5.2. How did you deal with your sick pigs?

\begin{tabular}{|c|c|}
\hline Report to vet. authority & \\
\hline Treatment? & \\
\hline If yes, what medicine? & ...…… \\
\hline Sell to the butcher & \\
\hline Other (details) & \\
\hline
\end{tabular}

\begin{tabular}{|c|c|c|c|}
\hline \multirow{3}{*}{5.3} & \multirow{3}{*}{$\begin{array}{l}\text { During April to June period did you buy in } \\
\text { from livestock markets or other places? }\end{array}$} & \multirow{4}{*}{$\begin{array}{l}\text { Pig } \\
\text { Other animals.......... } \\
\text { Yes }\end{array}$} & Number \\
\hline & & & ….......... \\
\hline & & & \\
\hline \multirow{3}{*}{5.4} & \multirow{3}{*}{$\begin{array}{l}\text { If yes, did you separate the newly bought } \\
\text { animal from the herd in an isolate pen? }\end{array}$} & & \\
\hline & & No & \\
\hline & & How long? Details & \\
\hline
\end{tabular}




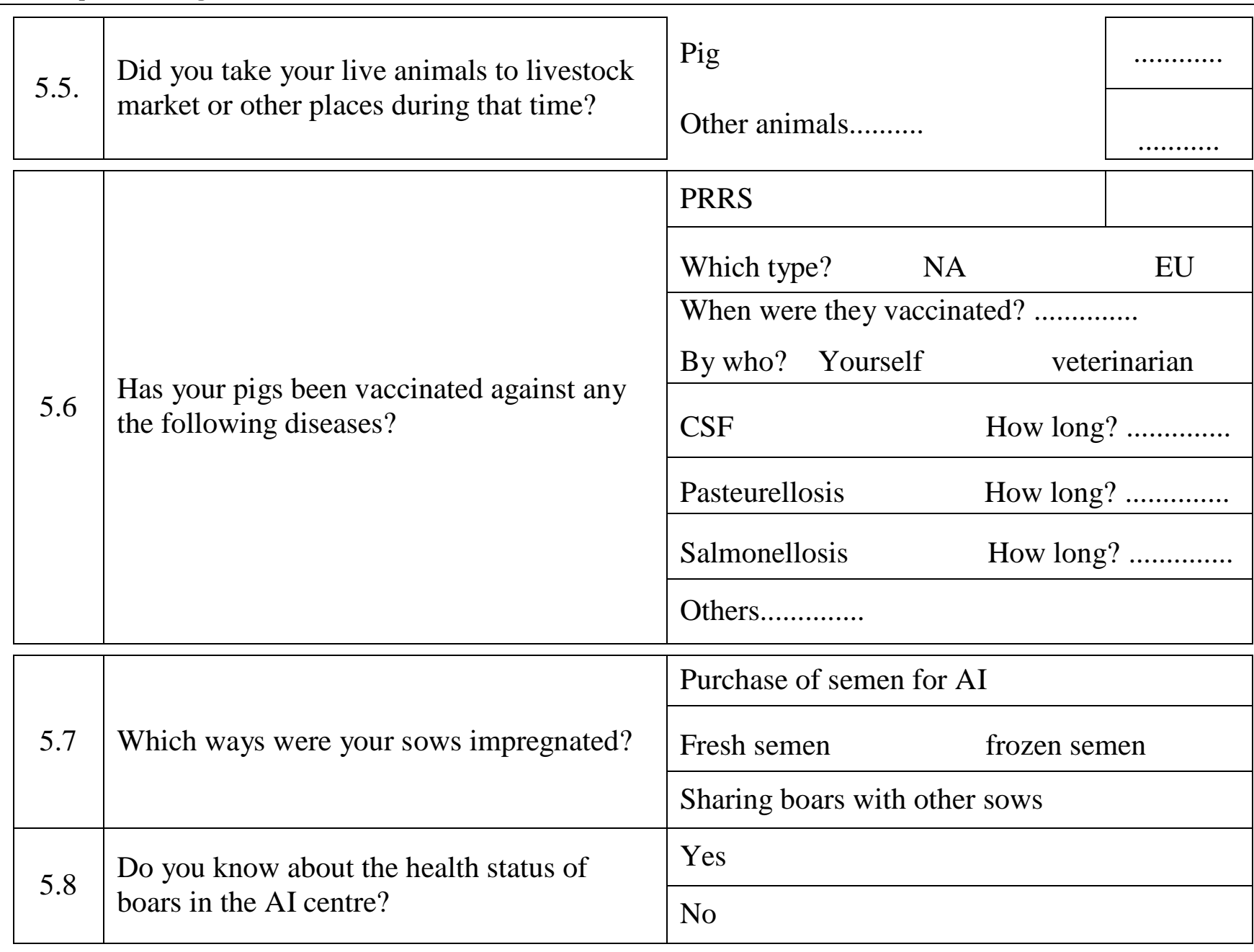

5.9. During the April-June period, who visited your pig pen or your pig farm?

\begin{tabular}{|c|c|c|c|c|c|}
\hline \multicolumn{2}{|l|}{ Family member } & \multirow[b]{2}{*}{ monthly } & \multicolumn{2}{|c|}{ Veterinary practitioner } & \multirow[b]{2}{*}{ monthly } \\
\hline How often: Daily & weekly & & How often: & weekly & \\
\hline \multicolumn{2}{|l|}{ Pig trader } & & \multicolumn{2}{|l|}{ Neighbours } & \\
\hline How often: Daily & weekly & monthly & How often: Daily & weekly & monthly \\
\hline \multicolumn{2}{|l|}{ Feed supplier } & & \multicolumn{2}{|l|}{ Other (details) } & .......... \\
\hline How often: Daily & weekly & monthly & How often: Daily & weekly & monthly \\
\hline \multicolumn{3}{|c|}{$\begin{array}{l}\text { 5.10. During April-June period, Did you keep other } \\
\text { animals? (dog, cat, chicken, duck) }\end{array}$} & \multicolumn{3}{|c|}{$\begin{array}{l}\text { 5.11. During that time, did you see the wild } \\
\text { animals like rat, wild birds }\end{array}$} \\
\hline \multicolumn{2}{|l|}{ Yes } & & Yes & & \\
\hline \multicolumn{2}{|l|}{ Which animal? } & ............ & Which animal? & & \\
\hline \multicolumn{3}{|c|}{ Were they totally confined? } & Rat & & \\
\hline \multicolumn{2}{|l|}{ Yes } & & Wild bird & & \\
\hline \multicolumn{2}{|l|}{ No } & & Which bird? Details & & \\
\hline
\end{tabular}


Partially confined

5.12. Were any of these animals sick during this period?

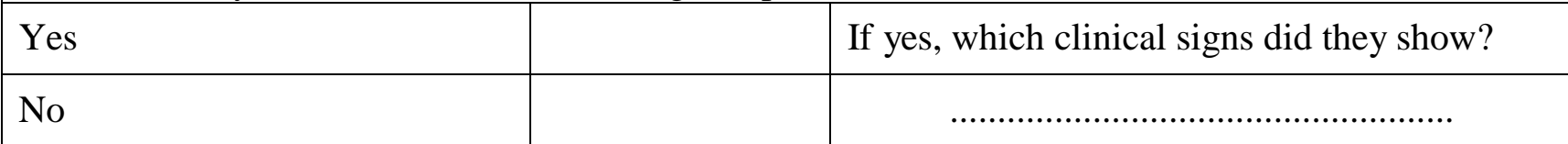

5.13. During April-June period, Did you go to the place where outbreak was occurring?

Yes

Which place?

5.15. During April-June period, Did you see anybody buy diseased pigs in your residence? Yes

No

No idea
5.14. During that time, did you sell your pig to the butcher or dealer?

Yes

When?

5.16. During that time, did you see anybody in your residence sell the diseased pigs?

Yes

No

No idea

An additional question was supplement to the questionnaire during survey.

Which source of water did you use for raising pig?

Pipe water

Underground

water From

irrigation system

THANK YOU FOR YOUR CO-OPERATION IN THIS INVESTIGATION.

INTERVIEWER 